\title{
SEGMENTATION OPTIMIZATION VIA RECOGNITION OF THE PSE-NSR-ED2 PATTERNS ALONG WITH THE SCALE PARAMETER IN OBJECT-BASED IMAGE ANALYSIS
}

\author{
Y. Liu a, b, *, Y-D. Zhang a , Z. Huang a, M-M. Wang a , D. Yang a H-M. Ma a , Y-X. Zhang a , Y-F. Li a , H-W. Li a , X-G. Hu ${ }^{a}$ \\ ${ }^{a}$ College of Earth and Environmental Sciences, Lanzhou University, Lanzhou 730000, China -(liuy, zhangyd5)@1zu.edu.cn; \\ huangzh07@126.com; lystwmm@163.com; 764423127@qq.com; (mahm2014, zhangyongxu14, liyp13)@1zu.edu.cn; \\ lihongweibang@126.com; huxg13@1zu.edu.cn \\ ${ }^{\mathrm{b}}$ Key Laboratory of Western China's Environmental Systems (Ministry of Education), Lanzhou University
}

KEY WORDS: object based image analysis; discrepancy measure; image segmentation; optimization; data stack; scale parameter

\begin{abstract}
:
To create image objects for subsequent classification in object-based image analysis, an optimal segmentation threshold (OST) is a pre-requisite for image segmentation. However, an OST is practically acquired by assessing and ranking an exhaustive segment data stack constructed after foregoing image segmentation. In this paper, we propose an iterative exploration method via recognition of the Euclidean distance 2 (ED2)-scale parameter (SP) pattern with the least five tiles of segment data stack in each cycle among the Potential Segmentation Error (PSE)-Number-of-Segments Ratio (NSR)-ED2 patterns along with SP. We conducted two experiments. In the first experiment, we validated the general italic U-shaped ED2-SP pattern by constructing exhaustive segment data stacks and corresponding segment data stacks. In the second experiment, we adopted the proposed iterative exploration method for OST selection based on the ED2-SP pattern with respect to five equal-spacing SPs in each cycle. The bottom of the pattern was persistently approached by constructing updated segment data stacks and corresponding segment data stacks with five dynamically adjusted tiles. Our results showed that the PSE-NSR-ED2 discrepancy measure system is advantageous to OST selection.
\end{abstract}

\section{INTRODUCTION}

Image segmentation, as the first critical step in the object-based image analysis (OBIA) procedure, divides an image into nonoverlapping segment datasets and assigns each pixel within an image to an inner-homogeneous segment (Navulur, 2007; Tilton et al., 2016). In general, image segmentation is performed by predefining a threshold to assess the maximum allowable spectral or spatial heterogeneity within image objects (Baatz et al., 2008; Baatz and Schäpe, 2000; Blaschke, 2010; Blaschke et al., 2014; Ming et al., 2015). However, selecting an optimal threshold is often a tedious trial-and-error process (Castilla and Hay, 2008; Meinel and Neubert, 2004). In addition, the optimal threshold changes with respect to images and land cover types.

The exhaustive method is often employed for selecting an optimal segmentation threshold (OST) based on quantitative assessment and ranking with respect to an exhaustive segment data stack (Arvor et al., 2013; Im et al., 2014; Marpu et al., 2010; Myint et al., 2011). An exhaustive segment data stack consists of tiles of segment datasets, and each tile is related to a threshold. Reference datasets play an important role in quantitative assessment of image segmentation. A corresponding segment dataset is a subset of segments for a tile in an exhaustive segment data stack, which does not cover the entire image but corresponds simply to a reference dataset spatially based on areal-overlap-based criteria (Clinton et al., 2010; Yang et al., 2015a). In general, each tile of corresponding segment data stacks exhibits a complexity of overlapping, undersegmentation, and oversegmentation, as compared with reference datasets. An optimal segment dataset (OSD) associated with an OST is qualified for the subsequent segment classification because it has the least undersegmentation and less oversegmentation as compared with a reference dataset (Castilla and Hay, 2008; Liu et al., 2012; Marpu et al., 2010; Radoux and Defourny, 2008; Weidner, 2008).

Many approaches have focused on developing automatic or semiautomatic procedures to obtain the OST based on goodness or discrepancy measures for segmentation assessment (Anders et al., 2011; Drăguţ et al., 2014; Martha et al., 2011; Rasi et al., 2011; Schultz et al., 2015; Smith, 2010). They are generally based on tiles of exhaustive segment data stacks associated with a wide range of segmentation thresholds and dense threshold intervals. A critical problem for segmentation optimization is to pre-set the minimal, maximal threshold, and the increment between the minimum to maximum. If the interval between the minimal and the maximal threshold is pre-set closely around the OST, the obtained OST can be a small number of tiles and the time spent is short. However, if the interval is given apart from the OST. The exhaustive method would be unsuccessful to select the OST. On the other hand, the OST could be omitted between the coarse increments of the threshold. It takes extremely long times to construct an exhaustive segment data stack with a great number of tiles associated with a wide range of segmentation thresholds and a dense threshold increment.

Discrepancy measures assess segment datasets by comparing reference polygons with corresponding segments (Albrecht et al., 2008; Lang et al., 2010; Marpu et al., 2010; Tiede et al., 2010; Zhang, 1996). Many discrepancy measures have been proposed for ranking and selecting the OSD and OST (Liu et al., 2012; Weidner, 2008; Yang et al., 2015a; Yang et al., 2015b; Yang et al., 2014; Zhang et al., 2015). Our previous studies 
have shown that the Potential Segmentation Error (PSE)Number-of-Segments Ratio (NSR)-Euclidean distance 2 (ED2) discrepancy measure system is credible (Liu et al., in press).

In this study, we propose an iterative exploration method for selecting the OST via recognition of the distribution patterns between the PSE-NSR-ED2 discrepancy measure and the scale parameter (SP). To verify this method, image segmentation is conducted on the basis of the fractal net evolution algorithm (FNEA) with a combined segmentation threshold consisting of three parameters: scale, shape, and compactness (Baatz and Schäpe, 2000; Burnett and Blaschke, 2003; Trimble, 2014). In this combination, the SP plays an important role in controlling the segment size. The other two parameters are weight factors varying between zero and one, and are mainly involved in improving the geometric characteristics of image objects. Each segment data stack is constructed and is employed for the optimal scale parameter (OSP) selection with a set of SP and a fixed combination of shape and compactness parameters. Thus, the OST selection turns to rank all the OSPs for the entire combinations of the shape and compactness parameters.

The remainder of this paper is organized into as follows. Section 2 explains an italic U-shaped ED2-SP pattern and an iterative transformation method of five dynamically adjusted equal-spacing SP values for automatically exploring the OSP. Sections 3 and 4 describe two experiments for validating the ED2-SP pattern and compare the results from these two method. Finally, Section 5 concludes the paper.

\section{METHOD AND DATA PREPARATION}

\subsection{Italic U-shaped ED2-SP pattern}

In this section, an italic U-shaped ED2-SP pattern is proposed based on the analysis of the PSE-SP, NSR-SP, and ED2-SP curves. Firstly, a power function between the average area of segments and the SP is suggested, given a numerical combination of the shape and compactness parameters. It follows by regularly variations of PSE and NSR along with the SP. The italic U-shaped ED2-SP pattern is therefore deduced.

In the PSE-NSR-ED2 discrepancy measure system, PSE is the ratio between the total area of the under-segments and the total area of the reference polygons, NSR is the absolute number difference between the reference polygons and the corresponding segments divided by the number of reference polygons, and ED2 is a Euclidean distance associated with PSE and NSR (Liu et al., 2012).

Here, we denote a reference dataset with $m$ reference polygons as $R=\left\{r_{i}: i=1,2, \ldots, m\right\}$ and a corresponding segment dataset with $n$ segments as $S=\{s j: j=1,2, \ldots, n\}$ based on one-side areal-overlap-based criteria. Thus, PSE, NSR, and ED2 can be expressed as

$P S E=\frac{\left|S_{k}-R_{i}\right|}{\left|R_{i}\right|} ; \quad N S R=\frac{\mathrm{abs}(m-n)}{m} ; E D 2=\sqrt{P S E^{2}+N S R^{2}}$

where

$\left|S_{k}-R_{i}\right|=$ total areas of the under-segments

$\left|R_{i}\right|=$ total areas of reference polygons

abs ()$=$ absolute value function.

A given image having a total area TA is divided into $n$ segment polygons; the average area of the segment polygons is given by:

$$
M A=\frac{T A}{n}
$$

where $\quad \mathrm{TA}=$ total number of pixels in the image.

As discussed in Section 3.2, our study verifies that, given a numerical combination of the shape and compactness parameter the mean area of segments increases approximately in the form of a power function along with SP with positive proportional and power terms. Correspondingly, the number of segments decreases approximately in the form of a power function along with SP, but with a positive proportional term and a negative power term. Thus,

$$
M A=\alpha \cdot S P^{\beta} ; \quad n=\frac{T A}{\alpha} \cdot S P^{-\beta}
$$

where $\quad \mathrm{MA}=$ mean area of segments in a tile

$$
\alpha, \beta=\text { positive real number. }
$$

When the area and the number of reference polygons are constant, PSE and NSR vary approximately similar to MA and $\mathrm{N}$, respectively, along with $\mathrm{SP}$, with some bias due to differences of images and land cover types. When SP is small, the arithmetic relationship between the corresponding segments and the reference polygons is many-to-one, accordingly, PSE is close to zero and NSR is large. As shown in Equation (1), the maximal NSR is smaller than the ratio between $n$ and $m$. Along with the increased SP, MA increases and $\left|S_{k}-R_{i}\right|$ is gradually close to zero up to an interval where the arithmetic relationship becomes one-to-one and both PSE and NSR keep small (less than one at least). Beyond this interval, the area of the corresponding segments is larger than the area of the reference polygons and the difference tends to increase along with SP, and the increased undersegmentation and PSE follows the power function between MA and the SP. On the other hand, the arithmetic relationship between the corresponding segments and the reference polygons remains one-to-one, NSR tends to zero. Therefore, we establish an ED2-SP pattern showing the variation of ED2 in the form of the italic U-shaped curve along with SP (Figure 1).

It can be seen that ED2 varies regularly along with the SP axis. The lowest point for the minimal ED2 and OSP splits the italic $\mathrm{U}$-shaped pattern into the left and the right side. The italic Ushaped pattern is constructed because these two sides are asymmetrical. The right side generally has wider range of SP than the left side, provided by equal ED2 range. At the left side of this pattern, SP is smaller than the OSP, the italic U-shaped pattern is close to or overlaps with the decreasing NSR curve where PSE is very small. At the right side of the pattern, SP is larger than the OSP, the italic U-shaped curve is close or coincident to the PSE curve, where NSR is close to zero.

Owing to its regularity, it is possible to locate the OSP by recognition of this pattern. When ED2 is close to the minimum, the under-segment areas are very small comparing to the areas of reference polygons and the number of corresponding segments is close to that of reference polygons, therefore, PSE and NSR are less than one. In other word, ED2 associated with the OSP normally lies in a very limit interval and is small than one. 


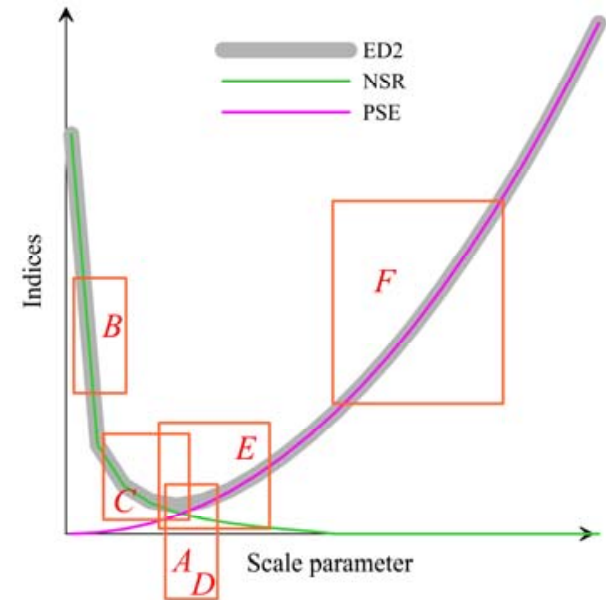

Figure 1. Italic U-shaped ED2-SP pattern under a numerical combination of the shape and compactness parameter. The ED2 curve coincides with the PSE and NSR curves at the two sides, respectively This italic U-shaped pattern is further divided into seven major cases, which are represented the ED2-SP pattern with five equal-spacing SPs in Figure 2 (from panel $A$ to $F$ ).

A critical aspect to consider in the OSP selection is that the pattern could vary with respect to the characteristics of remotely sensed imagery and land cover types. There is no universal coefficient setting for the ED2-SP pattern. The setting is unknown until a segment data stack and corresponding segment data stack are constructed. As indicated before, constructing an exhaustive segment data stack and corresponding segment data stack is bound to time-consuming. Alternatively, we proposed an iterative method for pattern recognition by constructing least five tiles of segment data stack and corresponding segment data stack for a combination of shape and compactness parameters. Provided by the entire OSP sets with respect to all the combinations of the shape and compactness parameters, the OST is selected by ranking the OSP set.

\subsection{Iterative exploration method for OSP selection via recognition of the ED2-SP pattern with five tiles of a segment data stack}

Graphically depicting a precise italic U-shaped pattern requires many ED2-SP dots. Reducing the dot number implies decreasing the tiles of segment data stack and the time spent for constructing the data stack. As performed in Section 4, five ED2-SP dots associated with five equal-spacing SPs for constructing a segment data stack and corresponding data stack are feasible to recognize the pattern in an iterative procedure.

The basic step is to construct a segment data stack with five tiles associated with five equal-spacing SP. Given a preliminary setting of the minimal and maximal SP, the other three SP can be interpolated equally between the ranges. Then, the PSENSR-ED2 measures are obtained and the ED2-SP pattern with five dots is constructed for each corresponding segment data stack.

Based on the pattern recognition with respect to the five ED2SP sets, a transformation to obtain updated five equal-spacing $\mathrm{SP}$ is conducted iteratively until the minimal ED2 at the bottom of the italic U-shaped pattern is found.
The ED2-SP pattern shown in Figure 1 represents only a general pattern. The actual pattern is offset owing to the differences with respect to images and reference datasets. Our study demonstrated that the variations of the five ED2 associated with the five equal-spacing SP can be classified into 17 cases. The pre-set minimal and maximal SP could be related to one of these cases. Six of them are the most important ones. They are shown as typical parts of the italic U-shaped ED2-SP pattern in Figure 1 and labelled also as panels $A-F$ in Figure 2. Case A could be prior to explore the OSP. However, it sometimes happens at the right side of the italic U-shaped pattern relevant to ED2s larger than 1. Case B is related to the left side of the pattern. Cases C-F happen at the bottom and the right side of the pattern. The other 11 Cases from $\mathrm{G}$ to $\mathrm{Q}$ can be meet mostly at the right side of the pattern relevant to ED2s larger than 1 .
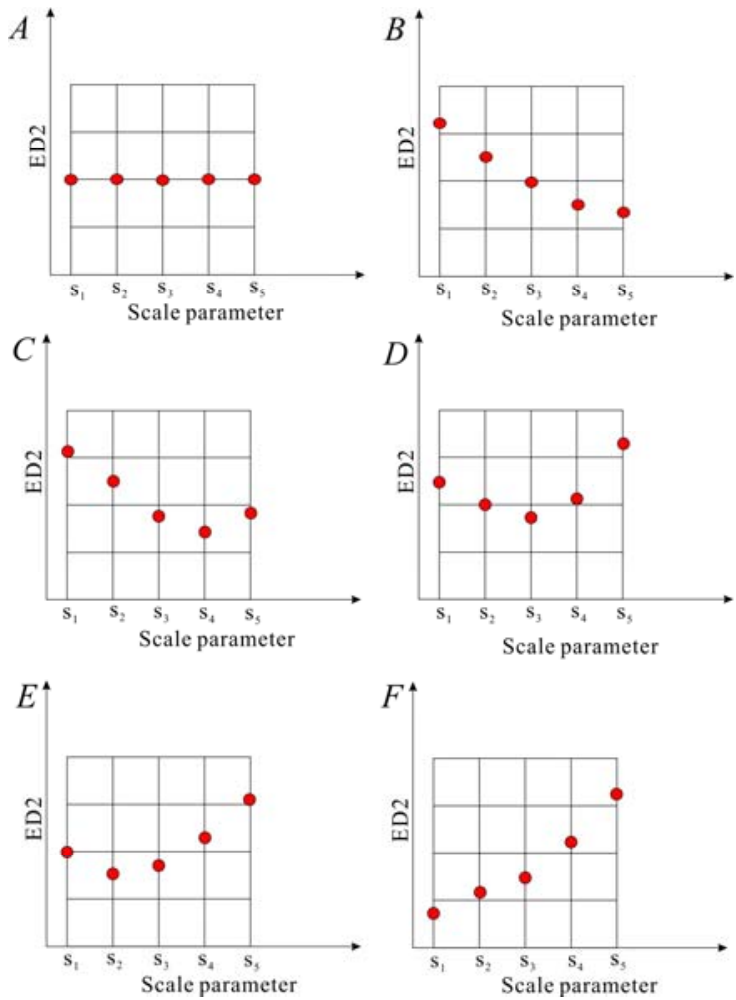

Figure 2. Major six cases of ED2 distribution associated with five equal-spacing SP.

To reach the target OSP interval as the case $A$ and $D$, a transformation is necessary to reset the SP range for the other cases based on their locations in the italic U-shape ED2-SP pattern. The basic transformations are one-dimensional zooming in, zooming out, left shifting, and right shifting along with the SP axis.

Initially, the SPs range $\left\{\mathrm{s}_{1}, \mathrm{~s}_{5}\right\}$ and the minimal interval between any two SPs, $d$ min, is predefined; $\mathrm{s}_{1}<\mathrm{s} 5$. To create a set of five SPs that are equally spaced from $\mathrm{s}_{1}$ to $\mathrm{s}_{5}$, we can sequentially interpolate the other three SPs, $\mathrm{s}_{2}, \mathrm{~s}_{3}$, and $\mathrm{s}_{4}$, as follows:

$d=\frac{1}{4}\left(s_{5}-s_{1}\right) ; \quad s_{3}=\frac{1}{2}\left(s_{1}+s_{5}\right) ; \quad s_{2}=\frac{1}{2}\left(s_{1}+s_{3}\right) ; \quad s_{4}=\frac{1}{2}\left(s_{3}+s_{5}\right)$ 
if the spacing between any pair of SPs is greater than the minimum, $d>d \min$. Otherwise, $\{\mathrm{s} 1, \mathrm{~s} 5\}$ need to be reset by increasing the spacing, $d$.

After setting these five SPs, s1-5, a segment data stack with five tiles is constructed. Further, a corresponding segment data stack is constructed with respect to a land cover type and the associated five ED2s are calculated.

The next step is to assess the pattern with the five ED2-SP dots in order to determine if one of the ED2s reaches the minimum and the OSP is coincident with the associated ED2. This assessment is performed case by case as follows.

In case $A$, the five ED2s associated with five equal-spacing SPs are equal or very close to each other. This case could occur in two situations. In the first situation, the five dots associated with the five ED2s really correspond to the bottom of the italic U-shaped pattern and indicate the minimal ED2. The OSP could be one of them. A further step is to confirm this situation by zooming out the five values by doubling the spacing, $d$, to update the five equal-spacing SPs:

$s_{1, \text { new }}=s_{1}-2 \cdot d ; \quad s_{2, \text { new }}=s_{1} ; \quad s_{3, \text { new }}=s_{3} ;$

$s_{4, \text { new }}=s_{5} ; \quad s_{5, \text { new }}=s_{5}+2 \cdot d$

where, S1-5, new represent the five updated SPs. If the updated pattern is coincident to the case $D$, as shown in Figure 2, the SP at $\mathrm{s}_{3, \text { new }}$ can be determined as the OSP.

In the second situation, these dots occur on the right side of the italic U-shaped pattern (Figure 1), associated with the ED2s larger than one. Then, a left-shifting operation can be conducted to reset these five SPs by decreasing the SP spacing, $d$, by four units.

$s_{1, \text { new }}=s_{1}-4 \cdot d ; \quad s_{2, \text { new }}=s_{1}+d ; \quad s_{3, \text { new }}=\frac{1}{2}\left(s_{1}+s_{5}\right) ;$

$s_{4, \text { new }}=s_{5}-d ; \quad s_{5, \text { new }}=s_{1}$

After this left-shifting operation, the routine returns to a new iterative cycle to construct a new segment data stack with the new five SPs given by Equation (6) in order to extract the corresponding segment data stack, recalculate the five ED2s for subsequent exploration, and recognize the new ED2-SP pattern.

In case $B$, the five ED2s decrease sequentially along with increasing SP. The minimal ED2 for this ED2-SP pattern could be at the right side of $\mathrm{s}_{5}$ or around it. A right-shifting operation can be conducted, where the SPs are obtained by doubling the spacing $d$.

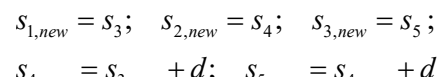

$s_{4, \text { new }}=s_{3, \text { new }}+d ; \quad s_{5, \text { new }}=s_{4, \text { new }}+d$

After this right-shifting operation, the routine returns to a new iterative cycle.

In case $C$, the minimal ED2 value occurs around S4. Furthermore, if $d \leq d \mathrm{~min}$ and the ED2 at $\mathrm{s} 4$ is smaller than one, then the SP can be determined as the OSP and the iterative operation terminates. If $d \geq d \mathrm{~min}$ and the ED2 at $\mathrm{s}_{4}$ is smaller than one, then the SP spacing is zoomed in to determine an improved estimation of the OSP. $d_{\text {new }}=\frac{1}{2} d ; \quad s_{1, \text { new }}=s_{2} ; \quad s_{5, \text { new }}=s_{4} ;$

$s_{2, \text { new }}=s_{1, \text { new }}+d ; \quad s_{3, \text { new }}=s_{2, \text { new }}+d ; \quad s_{4, \text { new }}=s_{5, \text { new }}-d ;$

Otherwise, if the ED2 at $\mathrm{s}_{4}$ is larger than one, then a rightshifting operation is conducted for decreasing the five SPs by one spacing $d$ :

$s_{1, \text { new }}=s_{2} ; \quad s_{2, \text { new }}=s_{3} ; \quad s_{3, \text { new }}=s_{4} ;$
$s_{4, \text { new }}=s_{5} ; \quad s_{5, \text { new }}=s_{5}+d$

In case $D$, the minimal ED2 occurs around $\mathrm{s}_{3}$. If $d \leq d \mathrm{~min}$ and the ED2 at $\mathrm{s}_{3}$ is smaller than one, then a zooming-in operation is conducted by halving the interval between $\mathrm{s} 1$ and $\mathrm{s} 5$, as shown in Equation (8). Otherwise, if the ED2 at $\mathrm{s}_{3}$ is greater than one, then a left-shifting operation is conducted to decrease these five SPs as shown in Equation (6).

In case $E$, the minimal ED2 occurs around $\mathrm{s}_{2}$. If $d \leq d \mathrm{~min}$ and the ED2 at s2 is smaller than one, then a zooming-in operation is conducted by halving the spacing between $\mathrm{s}_{1}$ and $\mathrm{s}_{5}$, as shown in Equation (8). Otherwise, if the ED2 at $\mathrm{s}_{2}$ is greater than one, then a left-shifting operation is conducted for decreasing the five SPs as shown in Equations (6).

In case $F$, these five ED2s increase sequentially along with the increasing SP. A left-shifting operation can be conducted for decreasing the SPs by doubling the spacing $d$.

$s_{5, \text { new }}=s_{3} ; \quad s_{3, \text { new }}=s_{1} ; \quad s_{4, \text { new }}=s_{2} ;$

$s_{5, \text { new }}=s_{3}-d ; \quad s_{1, \text { new }}=s_{2}-d$

For all the above cases with the left-shifting transformation, if the new $s_{1}$ is negative, then it must be reset to one and $s_{2-4}$ are recalculated, while s5 remains unchanged:

$s_{1, \text { new }}=1 ; \quad d=\frac{1}{4}\left(s_{5}-s_{1}\right) ; \quad s_{2, \text { new }}=\frac{1}{4} d ;$

$s_{3, \text { new }}=\frac{1}{2} d ; \quad s_{4, \text { new }}=\frac{3}{4} d ; \quad s_{5, \text { new }}=s_{5}$

\subsection{Data preparation}

Three types of images were principally employed in this study: QuickBird, WorldView II, and ALOS images. In addition, pansharpened multispectral data for the three images were constructed from all the multispectral bands and panchromatic band using the Gram-Schmidt algorithm. Therefore, we conducted our experiment using six sets of images, which, hereafter, are abbreviated for convenience as QB_MS, QB_sharpen, WV2_MS, WV2_ sharpen, ALOS_MS, and ALOS_sharpen.

Reference polygons were delineated for the six sets of images with regard to three types of land cover, namely farmland, rural housing, and water bodies, respectively. Each type of reference polygon was acquired using 30 samples for each image.

The computer employed in the following experiments had the following specification:

1) CPU: Intel ${ }^{\mathrm{TM}} \mathrm{Core}^{\mathrm{TM}} \mathrm{i} 7-3770,3.40 \mathrm{GHz}$;

2) Hard disk: Western Digital WD10 EALX-089BAO SATA $^{\mathrm{TM}}$, 1TB; 
3) Graphics adapter: NVIDIA ${ }^{\mathrm{TM}}, \mathrm{AMD}^{\mathrm{TM}}$ Radeon HD 7800 Series;

4) Operating System: Microsoft Windows 10 Professional, 64 bit.

\section{EXPERIMENT ONE: ED2-SP PATTERNS BASED ON EXHAUSTIVE METHOD}

The purpose of the experiment one is to verify (1) the power function between the average area of segments and the SP value; (2) the variations of PSE, NSR, and ED2 along with the SP based on the exhaustive segment data stacks from the six sets of images and corresponding segment data stacks associated with farmland, rural buildings, and water bodies.

\subsection{Construction of exhaustive segment data stacks and corresponding segment data stacks}

Image segmentation was conducted using the FNEA algorithm embedded in the eCognition ${ }^{\mathrm{TM}}$ software. To construct exhaustive segment data stacks, the minimal values, maximal values, and increments of SP were set to: 10, 100, and 5, respectively, for QB MS; 10, 190, and 20, respectively, for QB_sharpen; 5, 150, and 5, respectively, for WV2_MS; and 50, 350 , and 10, respectively, for WV2_sharpen. The minimal values, maximal values, and increments of both shape and compactness parameters were set to $0.1,0.9$, and 0.1 , respectively, for all the images. Therefore, there are 81 numerical combinations between the two parameter values for each SP, and the numbers of tiles for these six data stacks ranged from 810 to 2511 , as shown in Table 1 .

\begin{tabular}{|c|c|c|c|}
\hline Images & QB_MS & WV2_MS & ALOS_MS \\
\hline Image size & $1200 \times 1100$ & $1657 \times 1411$ & $290 \times 247$ \\
\hline $\begin{array}{c}\text { SP (min, max, } \\
\text { increment) }\end{array}$ & $10,100,5$ & $5,150,5$ & $10,150,10$ \\
\hline Number of tiles & 1539 & 2430 & 1215 \\
\hline$\alpha$ & $0.2918-1.1344$ & $0.1659-0.9975$ & $0.8487-6.0257$ \\
\hline$\beta$ & $1.7855-2.0144$ & $1.7745-2.1606$ & $1.8505-2.2414$ \\
\hline $\begin{array}{c}\text { Coefficient of } \\
\text { determinant }\end{array}$ & $0.9983-1.0000$ & $0.9793-0.9995$ & $0.9822-0.9991$ \\
\hline $\begin{array}{c}\text { Time spent } \\
\text { (hours) }\end{array}$ & 1.8407 & $\begin{array}{c}\text { S1: 6.0903; } \\
\text { S3: 7.3710; }\end{array}$ & 1.4805 \\
\hline Images & QB_sharpen & WV2_sharpen & $\begin{array}{c}\text { ALOS } \\
\text { sharpen }\end{array}$ \\
\hline Image size & $4800 \times 4400$ & $6630 \times 5644$ & $1067 \times 908$ \\
\hline $\begin{array}{c}\text { SP (min, max, } \\
\text { increment) }\end{array}$ & $10,190,20$ & $50,350,10$ & $50,650,50$ \\
\hline Number of tiles & 810 & 2511 & 1053 \\
\hline$\alpha$ & $0.2641-0.5544$ & $0.0778-1.0244$ & $0.7843-2.9019$ \\
\hline$\beta$ & $1.8218-2.1076$ & $1.6117-2.2615$ & $1.8175-2.0398$ \\
\hline $\begin{array}{c}\text { Coefficient of } \\
\text { determinant }\end{array}$ & $0.9961-0.9998$ & $0.9839-0.9997$ & $0.9932-0.9999$ \\
\hline $\begin{array}{c}\text { Time spent } \\
\text { (hours) }\end{array}$ & 17.6993 & $\begin{array}{c}\text { S1:108.6606; } \\
\text { S2:124.7088; } \\
\text { S3:123.7876 }\end{array}$ & 42.4262 \\
\hline Table 1. Statisics & for the prop & \\
\hline
\end{tabular}

Table 1. Statistics for the proportional and power coefficients, and the coefficient of determinant of the power function, and the time spent. The shape and compactness parameters are set from 0.1 to 0.9 at intervals of 0.1 with one accord.

Because of the large size of the WorldView II image $(6662 \times$ 5713 for eight bands and $26648 \times 22852$ for eight sharpened bands), it was divided into $(4 \times 4=) 16$ subsets. Only three subsets were selected in this experiment, based on the three dominant land cover types: farmland, rural housing, and water bodies. For convenience, they are abbreviated as FL, RH, and $\mathrm{WB}$, respectively.

\subsection{Results and discussion}

3.2.1 Power functions for the average area of segments and scale parameter

In this experiment, the power function for the average area of segments and SP was fitted with respect to each numerical combination of shape and compactness parameters. For this purpose, the average area of the segments in each data stack was calculated with respect to the SP.

The proportional and power coefficients $(\alpha$ and $\beta$ ) as well as the coefficients of determination of the power function, listed in Table 1, vary between 0.0778 and 6.0257 , and between 1.6117 and 2.2615, respectively, for the six types of images. Besides, all the coefficients of determinant take high values; the minimal coefficient is 0.9793 . It shows that the power function between the average area, number of segments, and SP is good for the given numerical combinations of shape and compactness parameter.

In addition, we conducted further data analysis using Chinese GF-1, Landsat 5 Thematic Mapper, and Landsat 8 Operational Land Imager data. Thus we confirmed that the power function is tenable with high coefficients of determination and various proportional and power coefficients.

\subsubsection{OST and time spent}

To extract corresponding segment data stacks, the reference datasets were employed for each land cover type with respect to the six sets of images. The overlapped, over-segmented, and under-segmented area were thus identified on the basis of the spatial overlap between the reference polygons and the corresponding segments. Furthermore, the PSE-NSR-ED2 discrepancy measure were calculated.

\begin{tabular}{|c|c|c|c|c|}
\hline $\begin{array}{c}\text { Image } \\
\text { sources }\end{array}$ & $\begin{array}{l}\text { Land } \\
\text { cover }\end{array}$ & $\begin{array}{c}\text { OST (scale, } \\
\text { shape, } \\
\text { compactness) }\end{array}$ & $\begin{array}{l}\text { Minimal } \\
\text { ED2 }\end{array}$ & $\begin{array}{c}\text { time spent } \\
\text { (hours) }\end{array}$ \\
\hline \multirow{3}{*}{ QB_MS } & FL & $15,0.3,0.9$ & 0.261 & 14.2382 \\
\hline & $\mathrm{RB}$ & $15,0.9,0.1$ & 0.368 & 15.0932 \\
\hline & WB & $50,0.5,0.7$ & 0.023 & 10.8182 \\
\hline \multirow{3}{*}{ WV2_MS } & FL & $25,0.6,0.4$ & 0.117 & 20.2653 \\
\hline & $\mathrm{RB}$ & $25,0.6,0.5$ & 0.292 & 21.5460 \\
\hline & WB & $150,0.3,0.6$ & 0.017 & 23.0166 \\
\hline \multirow{3}{*}{$\begin{array}{c}\text { ALOS_- } \\
\text { MS }\end{array}$} & FL & $30,0.2,0.3$ & 0.345 & 8.5680 \\
\hline & RB & $30,0.5,0.2$ & 0.264 & 7.8930 \\
\hline & WB & $20,0.4,0.7$ & 0.271 & 7.8930 \\
\hline \multirow{3}{*}{$\begin{array}{c}\mathrm{QB} \\
\text { sharpen }\end{array}$} & FL & $70,0.1,0.8$ & 0.211 & 24.2243 \\
\hline & $\mathrm{RB}$ & $90,0.6,0.3$ & 0.324 & 23.9993 \\
\hline & WB & $190,0.6,0.7$ & 0.039 & 24.8993 \\
\hline \multirow{3}{*}{$\begin{array}{l}\text { WV2_- } \\
\text { sharpen }\end{array}$} & FL & $130,0.2,0.5$ & 0.107 & 124.0056 \\
\hline & RB & $90,0.3,0.4$ & 0.236 & 153.3053 \\
\hline & WB & $320,0.7,0.3$ & 0.076 & 157.9651 \\
\hline \multirow{3}{*}{$\begin{array}{l}\text { ALOS_ } \\
\text { sharpen }\end{array}$} & FL & $100,0.1,0.7$ & 0.246 & 47.6912 \\
\hline & $\mathrm{RB}$ & $100,0.3,0.9$ & 0.454 & 47.6912 \\
\hline & WB & $50,0.3,0.9$ & 0.123 & 52.9562 \\
\hline
\end{tabular}

Table 2. OST selection and time spent based on the exhaustive method.

The OSTs were selected by ranked ED2s for each corresponding segment data stack. Table 2 lists the OST, minimal ED2, tiles in each segment data stack, and time spent with respect to image and land cover. The OST apparently varies with land cover. Notably, the OST for water bodies is 
mainly characterized by larger SP and smaller ED2 than those for farmland and rural housing.

3.2.3 Assessment of the italic U-shaped ED2-SP pattern Based on the corresponding segment data stacks, the ED2-SP pattern was assessed with respect to PSE-SP and NSR-SP curves. In total, there were 1458 sets of curves for the six images of the three land cover types, associated with 81 combinations of shape and compactness parameters $(6 \times 3 \times 9 \times$ 9). Figure 3 shows that a set of PSE, NSR, and ED2 varies along with SP for farmland from the WV2_MS image, where shape and compactness parameters are set to 0.5 and 0.1 , respectively. It is characterized by a trend similar to the general italic U-shaped ED2-SP pattern with some modifications. PSE is smaller than 0.5 until SP is less than or equal to 25 ; then, it increases along with SP. In the interval of $130-135$ for SP, the curve becomes horizontal and PSE do not change. NSR decreases drastically and monotonically from a very large number associated with SP of 5 to zero associated with SP of 55 When SP is greater than 55, NSR is zero. Thus, the number of reference polygons equals the number of corresponding segments. The ED2-SP curve represents a combined form of the PSE-SP and NSR-SP with the italic U-shaped pattern. When SP is smaller than 25 , the curve coincides with the part for NSR; when SP is larger than 55, the curve coincides with the part for PSE. The minimal ED2 is very small and appears in the interval of $25-55$ for SP.

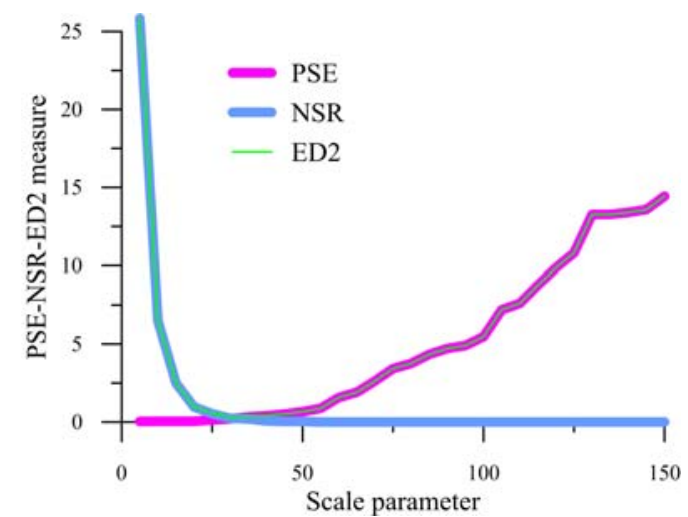

Figure 3. Variations in the PSE-NSR-ED2 along with SP for farmland from WV2 MS. The ED2 curve is shown by the thin green line superimposed on the other two curves (thick pink and blue lines) to indicate the local coincident relations among them.

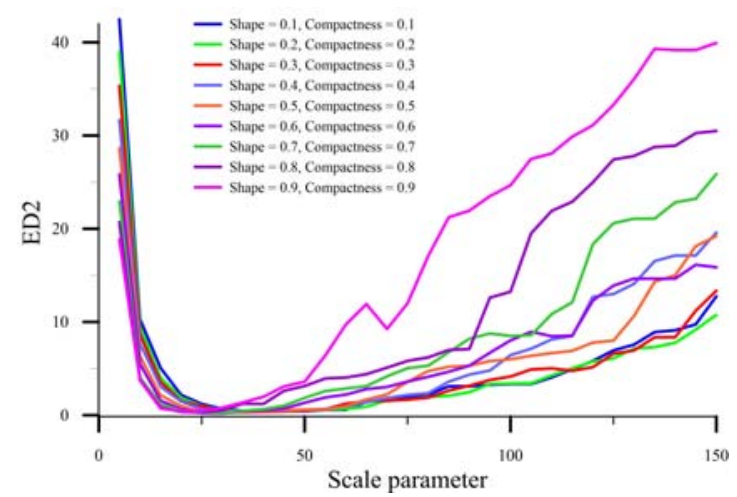

Figure 4. Italic U-shaped ED2-SP pattern obtained by some combinations of shape and compactness parameters.

Figure 4 shows the variations in ED2 along with SP with respect to nine sets of different shape and compactness parameters from the WV2_MS image for farmland. Some common trends for these nine curves are apparent. The ED2 variations along with SP present prominently diverse and is not well preserved as shown the general pattern in Section 2.1. At the left side of this curve, it decreases monotonically along with SP. However, the variations become complex in the right side, especially those associated with higher values of shape and compactness parameters. In some parts, ED2 are unchanged along with SP. When SP is larger than 0.6 , the monotonically increasing curve is reversed and local valleys appear sometimes. These abnormal changes occur when ED2 is larger than 1.0. All these variations are considered in the automatic exploration method, as discussed in Section 2.2.

\section{EXPERIMENT TWO: ITERATIVE OSP EXPLORATION METHOD BASED ON FIVE DOTS ED2-SP PATTERN}

The proposes of the experiment two are to verify the proposed iterative exploration method for OSP selection based on the five dots ED2-SP pattern and to compare the method with the exhaustive method.

\subsection{Construction of preliminary segment data stack with five tiles}

The second experiment was conducted using the same sets of image and reference datasets, as those employed in the first experiment, but with iterative exploration of the OSP with five equal-interval SPs. In this experiment, the preliminary minimal and maximal SPs were set to 10 and 50, respectively, for six images types and three land cover types, except for water bodies with respect to QB_sharpen (110 and 150, respectively), and for water bodies with respect to WV2_sharpen (410 and 450 , respectively). These values can be adjusted dynamically in an iterative routine to locate their position in the italic U-shaped ED2-SP patterns. Correspondingly, the minimal value, maximal value, and increment of both the shape and compactness parameters were set to $0.1,0.9$, and 0.1 , respectively. To recognize the ED2-SP pattern, both the minimum value and increment of SP were set to one, and two ED2s were regarded as equal if the difference between them is small than 0.0001 .

\subsection{Comparison between the exhaustive method and the iterative exploration method}

The results of the iterative exploration method are presented in Table 3. Comparing these results with those of the exhaustive method (shown in Table 2), it is clear that four sets of OSTs are consistent: the scale, shape, and compactness parameters are of $50,0.5$, and 0.7 for water bodies from QB MS; 25, 0.6, and 0.4 for farmland, and 25, 0.6, and 0.5 for rural housing from WV2_MS; and 70, 0.1, and 0.8 for farmland from QB_sharpen. More sets are close to the SP because the SP increment is set to one, which is smaller than that set in the exhaustive method, along with an improved ED2. Notably, the OST selected for water bodies based on WV2_MS and QB sharpen by the iterative exploration method lies outside of the pre-set intervals employed in the exhaustive method particularly for a larger SP (158 and 202, respectively).

The time spent changes from 7.425 hours for rural housing based on WV2_MS to 57.0375 hours for water bodies based on WV2_sharpen. Notably, the time spent does not decrease in all the cases, because the computational SP increment is set to one in order to track the italic U-shaped ED2 pattern. Provided by such a SP increment, the selection would take an extremely 
long time when employing the exhaustive method. In particular, the time spent is extremely long, e.g., for water bodies from WV2_MS, for farmland, rural housing, and water bodies from QB_sharp and WV2_sharpen, and for farmland and rural housing from ALOS_sharpen, because the initial setting of the minimum and maximum SP values exceed the results. Therefore, the initial setting of the SP for automatic exploration is critical for efficiently reducing the time spent.

\begin{tabular}{|c|c|c|c|c|c|}
\hline $\begin{array}{l}\text { Image } \\
\text { source }\end{array}$ & $\begin{array}{l}\text { Land } \\
\text { cover }\end{array}$ & $\begin{array}{l}\text { Number } \\
\text { of tiles }\end{array}$ & $\begin{array}{l}\text { OST (scale, } \\
\text { shape, } \\
\text { compactness) }\end{array}$ & ED2 & $\begin{array}{c}\text { Time } \\
\text { spent } \\
\text { (hours) }\end{array}$ \\
\hline \multirow{3}{*}{ QB_MS } & FL & 1296 & $17,0.1,0.1$ & 0.240 & 9.4500 \\
\hline & $\mathrm{RH}$ & 972 & $24,0.7,0.5$ & 0.353 & 8.1000 \\
\hline & WB & 1296 & $50,0.5,0.7$ & 0.023 & 10.8000 \\
\hline \multirow{3}{*}{$\begin{array}{c}\mathrm{WV}_{2}^{-} \\
\mathrm{MS}\end{array}$} & FL & 1053 & $25,0.6,0.4$ & 0.117 & 8.7750 \\
\hline & $\mathrm{RH}$ & 891 & $25,0.6,0.5$ & 0.292 & 7.4250 \\
\hline & WB & 2430 & $158,0.3,0.6$ & 0.017 & 20.2500 \\
\hline \multirow{3}{*}{$\begin{array}{c}\mathrm{ALOS}_{-} \\
\mathrm{MS}\end{array}$} & FL & 972 & $27,0.1,0.1$ & 0.327 & 8.1000 \\
\hline & RH & 1134 & $35,0.4,0.5$ & 0.225 & 9.4500 \\
\hline & WB & 1134 & $28,0.3,0.3$ & 0.207 & 9.4500 \\
\hline \multirow{3}{*}{$\begin{array}{c}\mathrm{QB}_{-} \\
\text {sharpen }\end{array}$} & FL & 1539 & $70,0.1,0.8$ & 0.211 & 41.6813 \\
\hline & $\mathrm{RH}$ & 1863 & $91,0.6,0.3$ & 0.308 & 50.4563 \\
\hline & WB & 1296 & $202,0.6,0.7$ & 0.030 & 35.1000 \\
\hline \multirow{3}{*}{$\begin{array}{c}\text { WV2_ } \\
\text { sharpen }\end{array}$} & FL & 1944 & $130,0.2,0.5$ & 0.107 & 52.6500 \\
\hline & $\mathrm{RH}$ & 1782 & $93,0.2,0.1$ & 0.228 & 48.2625 \\
\hline & WB & 2106 & $327,0.7,0.3$ & 0.076 & 57.0375 \\
\hline \multirow{3}{*}{$\begin{array}{l}\text { ALOS } \\
\text { sharpen }\end{array}$} & FL & 1782 & $96,0.1,0.7$ & 0.246 & 48.2625 \\
\hline & RH & 1863 & $82,0.3,0.7$ & 0.306 & 50.4563 \\
\hline & WB & 1215 & $41,0.3,0.7$ & 0.102 & 32.9063 \\
\hline
\end{tabular}

Table 3. OST selection and time spent for the automatic exploration method.

\section{CONCLUSIONS}

Based on the segment data stacks constructed with the fractal net evolution algorithm (FNEA) for image segmentation, this study verified that the mean area of segments increases approximately in the form of a power function along with SP, and the number of segments decreases approximately in the similar form of a power function. PSE and NSR vary approximately similar to the mean area and the number of segments, respectively, along with SP, with some bias due to differences of images and land cover types. Therefore, we establish an ED2-SP pattern showing the variation of ED2 in the form of the italic U-shaped curve along with SP. Owing to the regular variations of ED2 along with SP, the ED2-SP pattern is possible for recognition of the OSP. Furthermore, provided by the entire OSPs with respect to all the combinations of shape and compactness parameters, the OST is selected by ranking the OSPs.

In this study, we proposed and verified an iterative exploration method for OSP selection based on the italic U-shaped ED2-SP pattern. Given a preliminary range of SP, a segment data stack is constructed with five equal-spacing SPs, the PSE-NSR-ED2 measures are obtained and the five dots ED2-SP pattern with five pairs of ED2 and SP is constructed for the corresponding segment data stack associated with one land cover type. The variations of the five ED2 associated with the five equalspacing SP can be classified into 17 cases. Six of them are the most important. A linear transformation for the dynamical SP range is conducted iteratively until the minimal ED2 at the bottom of the italic U-shaped pattern is found. The bottom of the pattern was persistently approached by the transformation to obtain updated five equal-spacing SP and construction of updated segment data stacks and updated corresponding segment data stacks with five dynamically adjusted tiles.

The OSTs selected using the iterative exploration method are better than the exhaustive method. It is proved to be advantageous that an OSP is allowed for dense spacing SPs. Finally, an effective predefined SP range are advantageous for approaching the optimal SP value in the smallest time interval.

\section{ACKNOWLEDGEMENTS}

This research is supported by the National Science Foundation of China (NSFC) under grant 41271360.

\section{REFERENCES}

Albrecht, F., Lang, S., Hölbling, D., 2008. Spatial Accuracy Assessment of Object Boundaries for Object-Based Image Analysis, The International Archives of the Photogrammetry, Remote Sensing and Spatial Information Sciences, Vol. XXXVIII-4/C7.

Anders, N.S., Seijmonsbergen, A.C., Bouten, W., 2011. Segmentation optimization and stratified object-based analysis for semi-automated geomorphological mapping. Remote Sensing of Environment 115, 2976-2985.

Arvor, D., Durieux, L., Andrés, S., Laporte, M.-A., 2013. Advances in geographic object-based image analysis with ontologies: a review of main contributions and limitations from a remote sensing perspective. ISPRS Journal of Photogrammetry and Remote Sensing 82, 125-137.

Baatz, M., Hoffmann, C., Willhauck, G., 2008. Progressing from object-based to object-oriented image analysis, in: Blaschke, T., Lang, S. (Eds.), Object-based image analysis: spatial concepts for knowledge-driven remote sensing applications. Springer-Verlag, Berling.

Baatz, M., Schäpe, A., 2000. Multiresolution Segmentation: an optimization approach for high quality multi-scale image segmentation, in: Strobl, J., Blaschke, T., Griesebner, G. (Eds.), Angewandte Geographische Informations-Verarbeitung XIII, Wichmann, Heidelberg.

Blaschke, T., 2010. Object based image analysis for remote sensing. ISPRS Journal of Photogrammetry and Remote Sensing 65, 2-16.

Blaschke, T., Hay, G.J., Kelly, M., Lang, S., Hofmann, P., Addink, E., Feitosa, R.Q., van der Meer, F., van der Werff, H., van Coillie, F., 2014. Geographic object-based image analysis towards a new paradigm. ISPRS Journal of Photogrammetry and Remote Sensing 87, 180-191.

Burnett, C., Blaschke, T., 2003. A multi-scale segmentation/object relationship modelling methodology for landscape analysis. Ecological Modelling 168, 233-249.

Castilla, G., Hay, G.J., 2008. Image objects and geographic objects, in: Blaschke, T., Lang, S. (Eds.), Object-based image analysis: spatial concepts for knowledge-driven remote sensing applications. Springer-Verlag, Berling, Heidelberg, pp. 91-110.

Clinton, N., Holt, A., Scarborough, J., Yan, L., Gong, P., 2010. Accuracy Assessment Measures for Object-based Image 
Segmentation Goodness. Photogrammetric Engineering \& Remote Sensing 76, 289-299.

Drăguţ, L., Csillik, O., Eisank, C., Tiede, D., 2014. Automated parameterisation for multi-scale image segmentation on multiple layers. ISPRS Journal of Photogrammetry and Remote Sensing 88, 119-127.

Im, J., Quackenbush, L.J., Li, M., Fang, F., 2014. Optimum scale in object-based image analysis, in: Weng, Q. (Ed.), Scale Issues in Remote Sensing. John Wiley \& Sons, Inc, pp. 197-213.

Lang, S., Albrecht, F., Kienberger, S., Tiede, D., 2010. Object validity for operational tasks in a policy context. Journal of Spatial Science 55, 9-22.

Liu, Y., Bian, L., Meng, Y., Wang, H., Zhang, S., Yang, Y., Shao, X., Wang, B., 2012. Discrepancy measures for selecting optimal combination of parameter values in object-based image analysis. ISPRS Journal of Photogrammetry and Remote Sensing 68, 144-156.

Marpu, P.R., Neubert, M., Herold, H., Niemeyer, I., 2010. Enhanced evaluation of image segmentation results. Journal of Spatial Science 55, 55-68.

Martha, T.R., Kerle, N., van Westen, C.J., Jetten, V., Kumar, K.V., 2011. Segment Optimization and Data-Driven Thresholding for Knowledge-Based Landslide Detection by Object-Based Image Analysis. IEEE Transactions on Geoscience and Remote Sensing 49, 4928-4943.

Meinel, G., Neubert, M., 2004. A comparison of segmentation programs for high resolution remote sensing data, Commission VI on Proceeding to 20th ISPRS Congress, Istanbul.

Ming, D., Li, J., Wang, J., Zhang, M., 2015. Scale parameter selection by spatial statistics for GeOBIA: Using mean-shift based multi-scale segmentation as an example. ISPRS Journal of Photogrammetry and Remote Sensing 106, 28-41.

Myint, S.W., Gober, P., Brazel, A., Grossman-Clarke, S., Weng, Q., 2011. Per-pixel vs. object-based classification of urban land cover extraction using high spatial resolution imagery. Remote Sensing of Rnvironment 115, 1145-1161.

Navulur, K., 2007. Multispectral Image Analysis Using the Object-Oriented Paradigm. CRC Press, Taylor \& Grancis Group, Boca Raton.

Radoux, J., Defourny, P., 2008. Quality assessment of segmentation results devoted to object-based classification, in: Blaschke, T., Lang, S. (Eds.), Object-based image analysis: spatial concepts for knowledge-driven remote sensing applications. Springer-Verlag, Berling, Heidelberg, pp. 91-110.

Rasi, R., Bodart, C., Stibig, H.-J., Eva, H., Beuchle, R., Carboni, S., Simonetti, D., Achard, F., 2011. An automated approach for segmenting and classifying a large sample of multi-date Landsat imagery for pan-tropical forest monitoring. Remote Sensing of Environment 115, 3659-3669.

Schultz, B., Immitzer, M., Formaggio, A.R., Sanches, I.D.A., Luiz, A.J.B., Atzberger, C., 2015. Self-Guided Segmentation and Classification of Multi-Temporal Landsat 8 Images for Crop Type Mapping in Southeastern Brazil. Remote Sensing 7, 14482-14508.
Smith, A., 2010. Image segmentation scale parameter optimization and land cover classification using the Random Forest algorithm. Journal of Spatial Science 55, 69-79.

Tiede, D., Lang, S., Albrecht, F., Hölbling, D., 2010. Objectbased class modeling for cadastre-constrained delineation of geo-objects. Photogrammetric Engineering Remote Sensing 76, 193-202.

Tilton, J.C., Aksoy, S., Tarabalka, Y., 2016. Image segmentation algorithms for land categorization, in: Thenkabail, P.S. (Ed.). Taylor \& FRancis Group, LLC, Boca Raton, London, New York, pp. 317-342.

Trimble, 2014. eCognition Developer 9.0: User's Guide. Trimble Germany GmbH, Arnulfstrasse 126, D-80636 Munich, Germany.

Weidner, U., 2008. Contribution to the assessment of segmentation quality for remote sensing applications, The International Archives of the Photogrammetry, Remote Sensing and Spatial Information Sciences. ISPRS 2008, Beijing, pp. 479-484.

Yang, J., He, Y., Caspersen, J., Jones, T., 2015a. A discrepancy measure for segmentation evaluation from the perspective of object recognition. ISPRS Journal of Photogrammetry and Remote Sensing 101, 186-192.

Yang, J., He, Y., Weng, Q., 2015b. An Automated Method to Parameterize Segmentation Scale by Enhancing Intrasegment Homogeneity and Intersegment Heterogeneity. IEEE Geoscience and Remote Sensing.

Yang, J., Li, P., He, Y., 2014. A multi-band approach to unsupervised scale parameter selection for multi-scale image segmentation. ISPRS Journal of Photogrammetry and Remote Sensing 94, 13-24.

Zhang, X., Feng, X., Xiao, P., He, G., Zhu, L., 2015. Segmentation quality evaluation using region-based precision and recall measures for remote sensing images. ISPRS Journal of Photogrammetry and Remote Sensing 102, 73-84.

Zhang, Y.J., 1996. A survey on evaluation methods for image segmentation. Pattern Recognition 29, 1335-1346. 